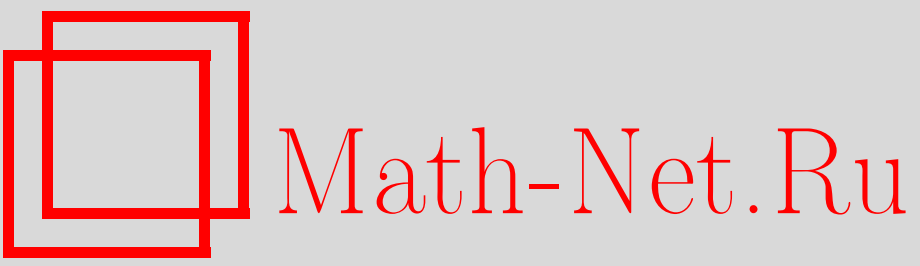

А. Ю. Брудный, Ю. А. Брудный, Совместные продолжения липшицевых функций, УМН, 2005, том 60, выпуск 6, 53-72

DOI: https://doi.org/10.4213/rm1676

Использование Общероссийского математического портала Math-Net.Ru подразумевает, что вы прочитали и согласны с пользовательским соглашением

http://www.mathnet.ru/rus/agreement

Параметры загрузки:

IP: 52.23 .180 .231

26 апреля 2023 г., 05:00:25 


\title{
СОВМЕСТНЫЕ ПРОДОЛЖЕНИЯ ЛИПШИЦЕВЫХ ФУНКЦИЙ
}

\author{
А. Брудный, Ю. Брудный
}

\begin{abstract}
Мы изучаем новый билипшицев инвариант $\lambda(M)$ метрических пространств $M$; конечность этой величины означает, что липшицевы функции на любом подмножестве $M$ могут быть линейно продолжены до функций на $M$, липшицевы постоянные которых увеличиваются на множитель $\lambda(M)$. Мы доказываем, что величина $\lambda(M)$ конечна для некоторых важных классов метрических пространств, включающих метрические деревья любой мощности, группы полиномиального роста, гиперболические группы в смысле Громова, некоторые классы римановых многообразий ограниченной геометрии и конечные прямые суммы любых комбинаций этих объектов. $\mathrm{C}$ другой стороны, мы приводим пример двумерного риманова многообразия ограниченной геометрии $M$ с $\lambda(M)=\infty$.

Библиографиия: 35 названий.
\end{abstract}

\section{СОДЕРЖАНИЕ}

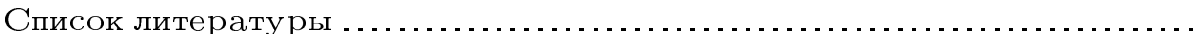

1. Введение. В настояший обзор включены недавние результаты авторов по задаче липшицева продолжения, подробно описанной ниже. Доказательства и некоторые дополнительные сведения можно найти в [10]-[12].

Пусть $(M, d)$ - метрическое пространство на множестве $M$ с метрикой $d$. В дальнейшем мы не указываем $d$ в обозначениях, если ее можно восстановить по контексту. Пространство вешественнозначных липшицевых функций $f$ на $M$ обозначим через $\operatorname{Lip}(M)$; это пространство снабжено стандартной полунормой

$$
L(f):=\sup _{m \neq m^{\prime}}\left\{\frac{\left|f(m)-f\left(m^{\prime}\right)\right|}{d\left(m, m^{\prime}\right)}\right\} .
$$

Подмножество $S \subset M$ будет рассматриваться как метрическое (под-) пространство, снабженное индуцированной метрикой $\left.d\right|_{S \times S}$. Следовательно, обозначения $\operatorname{Lip}(S)$ и $L(f)$ для $f \in \operatorname{Lip}(S)$ имеют смысл.

Совместное липшицево продолжение с $S$ на $M$ - это такой ограниченньй линейньй оператор $T: \operatorname{Lip}(S) \rightarrow \operatorname{Lip}(M)$, что

$$
\left.T f\right|_{S}=f, \quad f \in \operatorname{Lip}(S) .
$$

Исследование первого автора частично поддержано NSERC. 
Множество всех таких операторов $T$ обозначается через $\operatorname{Ext}(S, M)$, и (оптимальная) постоянная продолэсения определяется формулой

$$
\lambda(S, M):=\inf \{\|T\|: T \in \operatorname{Ext}(S, M)\} .
$$

(Мы полагаем $\lambda(S, M)=\infty$, если $\operatorname{Ext}(S, M)=\varnothing$.)

Определим теперь глобальную постоянную продолжсения формулой

$$
\lambda(M):=\sup _{S \subset M} \lambda(S, M) .
$$

Основной целью нашего исследования является следуюшая

ОСНОВНАЯ ЗАДАЧА. При каких геометрических свойствах $M$ величина $\lambda(M)$ конечна?

"Геометрическое" понимается здесь в смысле метрической (билипшицевой) геометрии, поскольку условие

$$
\lambda(M)<\infty
$$

инвариантно относительно билипшицевых гомеоморфизмов.

Эту задачу можно обобшить, заменяя постоянную продолжения $\lambda(M)$ величиной $\lambda(M, X)$, определяемой для банаховозначных липшицевых функций (со значениями в банаховом пространстве $X$ ) в точности так же, как и вьше. На самом деле это обобщение не слишком интересно. Действительно, легко видеть, что

$$
\lambda(M, X)=\lambda(M)
$$

для любого сопряженного банахова пространства $X$ (т.е. $X=Y^{*}$ для некоторого банахова пространства $Y$ ). Мы надеемся, что формула (5) справедлива и для произвольного $X$.

Сравним сформулированную вьше задачу с ее нелинейным вариантом, изучавшимся многими исследователями со времени публикации классических статей Киршбрауна и Макшейна в 1934 г. С этой целью мы введем постоянную нелинейного продолжения $\nu(M, X)$, заменяя линейные продолжения в определении $\lambda(M, X)$ нелинейными. Иначе говоря, определим $\nu(S, M, X), S \subset M$, как нижнюю грань постоянных $C$, для которых справедливо неравенство

$$
L(\tilde{f}) \leqslant C L(f),
$$

где $\widetilde{f}$ - липшицево продолжение $X$-значной липшицевой функции $f: S \rightarrow X$ на все $M$. Тогда положим

$$
\nu(M, X):=\sup _{S \subset M} \nu(S, M, X)
$$

и рассмотрим класс пространств $M$, для которых

$$
\nu(M, X)<\infty
$$

Ясно, что

$$
\nu(M, X) \leqslant \lambda(M, X),
$$

но различие между этими постоянными продолжения значительно. 
Рассмотрим сначала случай вещественнозначных функций $(X=\mathbb{R})$. Известная формула продолжения Макшейна [30] дает

$$
\nu(M)=1
$$

для всех метрических пространств $M$; с другой стороны, $\lambda(M)$ может быть бесконечной даже для двумерных римановых многообразий $M$ (поверхностей в $\mathbb{R}^{3}$ ), см. теорему 9 ниже.

Положим теперь $M=L_{2}[0,1]$ и $X=L_{p}[0,1]$. Есть только два случая, в которых

$$
(1=) \nu\left(L_{2}\right)=\nu\left(L_{2}, L_{p}\right)
$$

именно, из классической теоремы Киршбрауна [24] следует (9) при $p=2$, в то время как теорема Ароншайна и Паничпакди [2] дает этот результат при $p=\infty$. Для других значений $p$ результаты вьглядят следуюшим образом.

В фундаментальной статье Болла [3] доказано, что

$$
1<\nu\left(L_{2}, L_{p}\right)<\frac{6}{\sqrt{p-1}}
$$

при $1<p<2$, в то время как Наор [31] доказал, что $\nu\left(L_{2}, L_{p}\right)=\infty$ при $2<p<\infty$.

Но для постоянных линейного продолжения уже величина $\lambda\left(L_{2}\right)$ бесконечна, см. теорему 1 ниже; следовательно, $\lambda\left(L_{2}, X\right)=\infty$ для любого $X$.

Следует отметить несколько работ, посвященных верхним оценкам величины $\nu(M, X)$, но на самом деле связанных с постоянной линейного продолжения $\lambda(M, X)$.

В частности, Джонсон, Линденштраус и Шехтман [21] использовали модифицированньй метод продолжения Уитни [35], чтобы доказать, что для любого конечномерного банахова пространства $M$

$$
\lambda(M) \leqslant C \operatorname{dim} M,
$$

где $C$ - числовая постоянная ( $\approx 4000)$. Затем Матушек [29] доказал, что для любого метрического дерева $M$ имеем

$$
\lambda(M) \leqslant C
$$

с некоторой (не вычисленной) постоянной $C$.

Наконец, Ли и Наор [26] установили следующее важное неравенство:

$$
\lambda\left(l_{2}^{n}\right) \leqslant C \sqrt{n}
$$

с некоторой (не вычисленной) постоянной $C$.

Из (10)-(12) можно также получить соответствующие результаты для $X$-значных функций с любым банаховым пространством $X$ в качестве образа.

Понятие (и термин) совместного непрерьвного продолжения было введено в 1933 г. Борсуком [7]. Теорема продолжения Борсука была затем обобщена последовательно Какутани [22] и Дугунджи [17]. Теорема Дугунджи утверждает, что

для любого замкнутого подпространства $S$ метрического пространства $M$ существует оператор линейного продолжения с единичной нормой, действующий из $C_{b}(S, X)$ в $C_{b}(M, X)$.

Здесь $C_{b}(M, X)$ - банахово пространство $X$-значных непрерывных функций на $M$ с равномерной нормой, а $X$ - произвольное банахово пространство. 
Случай совместных липшицевых продолжений был впервые выделен Пелчинским в его книге 1968 г. [33]. Он написал, что “. . . наши знания о существовании операторов линейного продолжения для равномерно непрерьвных и липшищевых функций весьма недостаточны". Пелчинский заметил также, что для равномерно непрерьвных функций на бесконечномерных банаховых пространствах такие линейные операторы могут не сушествовать.

С тех пор и вплоть до 2003 г. активность в этом направлении была лишш спорадической. Сушественный прогресс в этой области был достигнут независимо в двух статьях, написанных в 2003 г. и использовавших совершенно разные методы, см. [10] и [26]. Подход авторов [10] был основан на комбинировании функционально-аналитических и геометрических методов, в то время как работа [10] основана на вероятностных рассуждениях (так называемом стохастическом метрическом разложении). Этот подход существенно отличается от подхода работы [26]. Поэтому мы приводим в настояшей работе главным образом результаты авторов, отсылая читателя к статьям [28], [3] и [26] по различным вопросам, связанным с вероятностным подходом к нелинейньм и линейным липшицевым продолжениям.

Даже это краткое перечисление показьвает, что сформулированная вьше задача, кажушаяся довольно скромной, оказьвается удивительно глубокой; упомянутые выше работы показывают, что ее решение требует значительного количества инструментов из разных областей анализа и геометрии.

2. Эта часть статьи имеет следующую структуру. Сначала мы вводим некоторые классы метрических пространств, имеющих интересуюшее нас свойство продолжения. Мы увидим, что многие важные метрические пространства, возникающие в анализе, геометрии и теории груп, обладают этим свойством. Затем мы формулируем некоторые обшие результаты, которые, в частности, обеспечивают конечность величины $\lambda(M)$ для этих классов метрических пространств.

Банаховы пространства. Простейший класс метрических пространств образуют банаховы пространства. Основной результат, доказанньй в нашей работе [11], имеет следуюший вид.

Tеорема 1. Пусть $X-$ банахово пространство. Тогда $\lambda(X)<\infty$ в том и только том случае, когда $\operatorname{dim} X<\infty$.

Это результат является следствием приведенной ниже теоремы 39, связьвающей линейные и нелинейные липшицевы постоянные.

Ввиду теоремы 1 , естественно ставится

ЗАДАЧА 2. Найти точную асимптотику величинь $\lambda\left(l_{p}^{n}\right)$ при $n \rightarrow \infty$.

Эта задача вьглядит довольно трудной. Следует заметить, что похожая задача о точной асимптотике постоянной $\lambda_{\text {conv }}\left(l_{p}^{n}\right)$ была решена, см. [10; теорема 2.18]. Здесь $\lambda_{\text {conv }}\left(l_{p}^{n}\right)$ определяется формулой $(3)$ при $M:=l_{p}^{n}$ и $S$, пробегаюшем все выпуклье подмножества в $l_{p}^{n}$. А именно, в этом случае справедливо следующее утверждение.

Теорема 3. Существует такая числовая постоянная $c_{0}>0$, что

$$
c_{0} \leqslant n^{-\left|\frac{1}{p}-\frac{1}{2}\right|} \lambda_{\text {conv }}\left(l_{p}^{n}\right) \leqslant 1
$$

Известные результаты, связанные с задачей 2 , приведены в следуюшей теореме. 
TEOPEMA 4. (а) При $p=1, \infty$ и при всех $n \geqslant 1$ имеем

$$
c_{0} \sqrt{n} \leqslant \lambda\left(l_{p}^{n}\right) \leqslant 24 n
$$

здесь $c_{0}-$ постоянная из теоремы 3.

(б) Для некоторых числовых постоянных $0<c_{1}<c_{2}$ и всех $n \geqslant 1$ имеем

$$
c_{1} n^{1 / 8} \leqslant \lambda\left(l_{2}^{n}\right) \leqslant c_{2} n^{1 / 2}
$$

Утверждение (а) вытекает из теоремы 3 (левое неравенство) и из следствия 2.26 в [10]. Правое неравенство в утверждении (б) уже приводилось в формуле (12) во введении, см. [26; теорема 5.1], в то время как левое неравенство доказано в [11]. На самом деле, из упомянутого вьше следствия 2.26 работы [10] вытекает, что для конечномерного банахова пространства $X$ имеем

$$
\lambda(X) \leqslant 24 \operatorname{dim} X,
$$

что улучшает приведенный вьше результат из [21] (см. (10)).

В связи с теоремами 3 и 4 мы можем высказать следующую гипотезу.

ГИПОТЕЗА 5. Имеет место әквивалентность

$$
\lambda\left(l_{p}^{n}\right) \sim \sqrt{n} \lambda_{\mathrm{conv}}\left(l_{p}^{n}\right)
$$

с постоянными әквивалентности, не зависящими от $n$.

Метрические пространства ограниченной геометрии. Для введения этого класса метрических пространств нам нужны следуюшие определения.

Пусть $\phi:\left(M_{1}, d_{1}\right) \rightarrow\left(M_{2}, d_{2}\right)$ - отображение одного метрического пространства в другое. Его липшицевой постоянной назьвается величина

$$
|\phi|_{\operatorname{Lip}\left(M_{1}, M_{2}\right)}:=\sup _{m^{\prime} \neq m^{\prime \prime}}\left\{\frac{d_{2}\left(\phi\left(m^{\prime}\right), \phi\left(m^{\prime \prime}\right)\right)}{d_{1}\left(m^{\prime}, m^{\prime \prime}\right)}\right\} .
$$

Отображение $\phi$ называется квазиизометрией, если $\phi$ - биекция и липшицевы постоянные для $\phi$ и $\phi^{-1}$ конечны. Мы назьваем $\phi C$-изометрией, если эти липшицевы постоянные ограничены постоянной $C \geqslant 1$ (и изометрией, если $C=1$ ).

ОПРЕДЕЛЕНИЕ $6 .^{1}$ Метрическое пространство $M$ называется пространством ограниченной геометрии, если сушествуют такие параметры $n \in \mathbb{N}, R, C>0$, что любой замкнутый шар радиуса $R$ в $M C$-изометричен замкнутому подмножеству $n$-мерного евклидова пространства.

Класс метрических пространств, удовлетворяюших определению 6, обозначим через $\mathscr{G}_{n}(R, C)$.

\footnotetext{
${ }^{1} \mathrm{Cp}$. соответствующее понятие для римановых многообразий в [15].
} 
Пример 7 . (а) Пусть $\Gamma=(V, E)$ - граф с множеством вершин $V$ и множеством ребер $E$ (мы рассматриваем только графы без петель и без кратных ребер). Напомним, что путь в Г, соединяющий вершины $v^{\prime}$ и $v^{\prime \prime}$,- это такая конечная последовательность вершин $v_{0}, v_{1}, \ldots, v_{n}$, что $v_{0}=v^{\prime}, v_{n}=v^{\prime \prime}$ и вершины $v_{i}$ и $v_{i+1}$ соединены (единственным) ребром (обозначаемым $\left[v_{i}, v_{i+1}\right]$ ). Отождествим согласованньм образом ребра $e$ с отрезками на числовой прямой с длинами $l_{e}>0$ и затем определим длину пути $p=v_{0}, \ldots, v_{n}$ формулой

$$
l(p):=\sum_{i=0}^{n-1} l_{\left[v_{i}, v_{i+1}\right]} .
$$

Это позволяет ввести расстояние $d_{\Gamma}\left(v^{\prime}, v^{\prime \prime}\right)$ между вершинами $v^{\prime}$ и $v^{\prime \prime}$ как нижнюю грань длин путей, соединяющих $v^{\prime}$ и $v^{\prime \prime}$. Мы можем естественным образом распространить это определение на случай внутренних точек $m^{\prime}, m^{\prime \prime}$ ребер.

Вообще говоря, величина $d_{\Gamma}\left(m^{\prime}, m^{\prime \prime}\right)$ может быть не определена, но $d_{\Gamma}$ становится метрикой, если $\Gamma-$ связный граф, т.е. любые две его вершины можно соединить путем. В этом случае мы будем назьвать $\left(\Gamma, d_{\Gamma}\right)$ метрическим графом. С топологической точки зрения, это метрическое пространство - одномерный $C W$-комплекс и $V$ - его 0-остов.

Приведенное выше "наивное" определение метрического граффа может быть сделано точным (см., например, [9; с. 6]).

Пусть теперь $\left(\Gamma, d_{\Gamma}\right)$ - метрический граф̆. Нетрудно проверить, что $\Gamma$ - метрическое пространство ограниченной геометрии тогда и только тогда, когда степени его вершин ${ }^{2}$ равномерно ограничены.

(б) Пусть $(R, g)$ - риманово многообразие с римановой метрикой $g$. Тогда любая гладкая кривая $\gamma:[0, a] \rightarrow R$ имеет длину $l(\gamma)$ (определяемую каноническим образом). С ее помощью можно определить расстояние $d_{g}\left(m^{\prime}, m^{\prime \prime}\right)$ между точками $R$ как нижнюю грань длин кривых, соединяюших $m^{\prime}$ и $m^{\prime \prime}$. Если $R$ - связное многообразие, то $\left(R, d_{g}\right)$ - метрическое пространство, и $d_{g}$ называется геодезической (внутренней) метрикой на $R$.

Легко видеть, что компактное риманово многообразие $(R, g)$, рассматриваемое как метрическое пространство $\left(R, d_{g}\right)$, есть пространство ограниченной геометрии. С другой стороны, (некомпактное) пространство Бельтрами-Лобачевского $\mathbb{H}^{n}$ тоже является пространством ограниченной геометрии.

ЗАмЕчАниЕ 8. Метрические пространства в этих примерах принадлежат классу метрических пространств путей. Напомним, что $(M, d)$ принадлежит этому классу, если расстояние между любыми двумя точками равно нижней грани длин кривых, соединяюших эти точки.

Вернемся к основной задаче для метрических пространств ограниченной геометрии. Используя теорему 1.6 статьи [26], легко доказать, что для $M \in \mathscr{G}_{n}(R, C)$ и для любого подпространства $S \subset M$ диаметра $\operatorname{diam} S \leqslant R$ вьполняется неравенство

$$
\lambda(S, M) \leqslant b C^{2} n,
$$

где $b$ - числовая постоянная. Казалось бы, на этом основании можно высказать следующее утверждение:

$$
M \in \mathscr{G}_{n}(R, C) \Longrightarrow \lambda(M)<\infty .
$$

Поразительным образом это неверно даже для метрических пространств малой размерности, как показывает следующий контрпример.

\footnotetext{
${ }^{2}$ Напомним, что $\operatorname{deg} v$ - число вершин, инцидентных вершине $v$.
} 
Теорема 9 (см. [10; предложение 10.1 и теорема 2.17]). (а) Cуществует метрический граф $\left(\Gamma, d_{\Gamma}\right)$, степени вершин которого не превосходят 3 , и такое подпространство $S_{0} \subset \Gamma$, что мнохсество операторов линейного продолхсения $\operatorname{Ext}\left(S_{0}, \Gamma\right)$ nусто. В частности, $\lambda(\Gamma)=\infty$.

(б) Существуют двумерное риманово многообразие $\left(R, d_{g}\right)$ ограниченной геометрии и его (метрическое) подпространство $S_{0} \subset R$, для которых выполняется то же утверждение: $\operatorname{Ext}\left(S_{0}, R\right)=\varnothing$.

Применяя теорему вложения Нэша, мы можем реализовать $R$ как $C^{\infty}$-поверхность в открытом шаре $\mathbb{R}^{3}$ (с римановой структурой, индуцированной структурой пространства $\mathbb{R}^{3}$ ). Что касается подмножества $S_{0}$ в обоих утверждениях, то оно определяется неконструктивно. А именно, основой построения является почти ортогональное разложение Кашина [23] пространства $l_{1}^{2 n}$ в два экземпляра пространства $l_{1}^{n}$. Насколько нам известно, все сушествуюшие доказательства теоремы Кашина основаны на тех или иных вероятностных рассуждениях.

Так как $\lambda(M)$ может оказаться бесконечным для метрических пространств ограниченной геометрии, мы сейчас введем достаточное условие его конечности. Формулируемые ниже результаты сводят задачу к аналогичной задаче для родственных метрических пространств с сушественно более простой структурой.

Для формулировки первого результата нам нужно следующее определение.

ОПРЕДЕлЕниЕ 10. Множество $\Gamma \subset M$ назьвается $R$-решеткой ${ }^{3}$, если семейство открытых шаров $\left\{B_{R / 2}(\gamma): \gamma \in \Gamma\right\}$ покрьвает $M$, в то время как шары $B_{c R}(\gamma), \gamma \in \Gamma$, попарно не пересекаются при некотором $c=c_{\Gamma} \in\left(0, \frac{1}{4}\right]$.

Сушествование $R$-решеток легко следует из леммы Цорна.

Теорема 11 [10; следствие 2.12]. Пусть $M \in \mathscr{G}_{n}(R, C)$. Величина $\lambda(M)$ конечна тогда и только тогда, когда существует такая $R$-решетка $\Gamma \subset M$, что

$$
\lambda(\Gamma)<\infty .
$$

Изучая примеры пространств ограниченной геометрии с $\lambda(M)=\infty$, можно заметить, что все $R$-решетки в этих пространствах имеют крайне иррегулярную структуру. С другой стороны, известные пространства этого типа с $\lambda(M)<\infty$ имеют равномерные $R$-решетки. Здесь термин "равномерная" означает, что для любого $r$ число точек в пересечении $\Gamma \cap B_{r}(\gamma)$ приблизительно одинаково для всех $\gamma \in \Gamma$. Точнее, сушествуют такая возрастаюшая функция $\psi_{\Gamma}: \mathbb{R}_{+} \rightarrow \mathbb{R}_{+}$и такая постоянная $c>0$, что для всех $r>0$ и $\gamma \in \Gamma$ имеем

$$
c \psi_{\Gamma}(r) \leqslant \operatorname{card}\left(\Gamma \cap B_{r}(\gamma)\right) \leqslant \psi_{\Gamma}(r) .
$$

Эти обсуждения приводят к следуюшей гипотезе.

ГиПотеЗА 12. Если Г - равномерная $R$-решетка в некотором метрическом пространстве $M$, то

$$
\lambda(\Gamma)<\infty .
$$

Следует отметить, что из приведенных ниже результатов следует, что гипотеза справедлива для функций $\psi_{\Gamma}$, удовлетворяюших условию удвоения:

$$
\sup _{t>0}\left\{\frac{\psi_{\Gamma}(2 t)}{\psi_{\Gamma}(t)}\right\}<\infty ;
$$

\footnotetext{
${ }^{3} \mathrm{~B}$ некоторых статьях множество Г называется отделимой сетью (separated net).
} 
то же верно для некоторых функций $\psi_{\Gamma}$ экспоненциального роста.

Несмотря на то, что каждое метрическое пространство имеет $R$-решетки, не все они имеют равномерные $R$-решетки. Если такая равномерная решетка существует в метрическом пространстве путей $M$ ограниченной геометрии, то справедливость гипотезы 12 означает, что $\lambda(M)<\infty$ (см. замечание 14 (в) ниже). Примером такой ситуации является связная группа Ли $G$ с геодезической метрикой $d_{g}$, определенной канонической левоинвариантной римановой структурой. Можно показать, что $\left(G, d_{g}\right)$ имеет равномерную $R$-решетку для некоторого $R>0$.

Наш следуюший результат связывает свойство конечности пространства $M$ (т.е. конечность величины $\lambda(M)-n p u м$. peд.) с аналогичным свойством некоторой конечно порожденной групшы изометрий $M$; эта группа рассматривается как метрическое пространство, снабженное расстоянием между словами.

Напомним основные понятия, входящие в последующую формулировку.

Пусть $G$ - группа с множеством образуюших $A$. Любой элемент $g \in G$ можно представить в виде слова $a_{1} a_{2} \ldots a_{r}$ (т.е. $g=a_{1} \cdots a_{r}$ ) с $a_{i} \in A \cup A^{-1}$. Длиной слова назьвается число сомножителей в нем. Расстояние между словами $d_{A}\left(g_{1}, g_{2}\right)$ для элементов $g_{1}, g_{2} \in G$ определяется как нижняя грань длин слов, представляюших элемент $g_{1}^{-1} g_{2}$. Группа $G$, наделенная расстоянием $d_{A}$, становится метрическим пространством.

Пусть $G$ - конечно порожденная группа, т.е. пусть множество $A$ конечно. Если $A^{\prime}$ - другое конечное множество образуюших для $G$, то пространства $\left(G, d_{A}\right)$ и $\left(G, d_{A^{\prime}}\right)$ квазиизометричны. Итак, в этом случае конечность величины $\lambda(G)$ не зависит от выбора $A$.

Пусть теперь $M$ - метрическое пространство, и пусть Iso $(M)$ - его группа изометрий. Предположим, что $G$ - подгруппа Iso $(M)$, действуюшая на $M$ (a) свободно, (б) собственно и (в) кокомпактно. Напомним смысл этих терминов.

(а) Для любого $m \in M$

$$
g(m)=m \Longrightarrow g=1 .
$$

(б) Для любого компактного множества $K \subset M$ множество

$$
\{g \in G: g(K) \cap K \neq \varnothing\}
$$

конечно.

(в) Существует такое компактное множество $K_{0} \subset M$, что

$$
M=\bigcup_{g \in G} g\left(K_{0}\right) .
$$

По лемме Ефремовича-Шварца-Милнора (см., например, [9; с. 140]) группа $G$, действуюшая на $M$ таким образом, конечно порождена, если $M$ - метрическое пространство путей (см. замечание 8 по поводу определения). Следовательно, в этом случае величина $\lambda(G)$ определена.

Теорема 13 [10; следствие 2.16]. Пусть $M$ - метрическое пространство путей ограниченной геометрии, и пусть $G \subset \operatorname{Iso}(M)$ действует на $M$ свободно, собственно и кокомпактно. Тогда

$$
\lambda(M)<\infty \Longleftrightarrow \lambda(G)<\infty
$$


ЗАмЕчАниЕ 14. (а) Теоремы 11 и 13 остаются справедливыми и для банаховозначных липшицевых функций со значениями в любом пространстве (не обязательно сопряженном).

(б) Доказательства этих теорем дают количественные оценки величины $\lambda(M)$ в терминах основных параметров, участвуюших в их формулировках.

(в) Теорема 13 есть следствие из теоремы 11. А именно, в условиях первой из этих теорем любая орбита $G(m)$ точки $m \in M$ является $R_{0}$-решеткой для некоторого $R_{0}>0$. Кроме того, метрическое пространство путей ограниченной геометрии принадлежит классу $\mathscr{G}_{n}(R, C)$ для любого $R>0$ и некоторого $C=C(R, M, n)$.

Конечно порожденные групшы. Теорема 13 сводит основную задачу для специальных метрических пространств ограниченной геометрии к той же задаче для конечно порожденных групп. Если бы гипотеза 12 была верна, то для любой конечно порожденной групшы $\Gamma$ постоянная продолжения была бы конечна, $\lambda(\Gamma)<\infty$. Ниже мы приведем результат (теорема 19), подтверждающий эту гипотезу.

A. Групшы полиномиального роста. Пусть $G$ - группа с множеством образуюших $A$. Такая группа назьвается группой полиномиального роста, если любой шар радиуса $R$ в $\left(G, d_{A}\right)$ содержит не более $c R^{n}$ элементов; здесь $c>0$ и $n \geqslant 0$-постоянные, не зависяшие от $R$.

Согласно теореме Громова, такая группа $G$ виртуально нильпотентна, т.е. сушествует такая нормальная нильпотентная подгруппа $N \subset G$, что $G / N$ - конечная группа. Пусть $\mu_{0}-$ считающая мера на $G$, т.е. $\mu_{0}(S):=\operatorname{card}(S)$. Басс [5] доказал, что сушествуют такие постоянные $c_{1}, c_{2}>0$, зависящие только от $\operatorname{card}(G / N)$, где $N-$ наибольшая нильпотентная подгруппа в $G$ без кручения, что для любого открытого шара $B_{R}(m) \subset\left(G, d_{A}\right)$

$$
c_{1} R^{Q} \leqslant \mu_{0}\left(B_{R}(m)\right) \leqslant c_{2} R^{Q} .
$$

Здесь $Q:=\sum_{h \geqslant 1} h r_{h}, r_{h}=$ ранг без кручения группы $G_{h} / G_{h+1}$ и $G=G_{1} \supseteq G_{2} \supseteq \ldots$ - нижний центральньй ряд группы $G$.

Из этого неравенства следует, что $\mu_{0}$ - мера со свойством удвоения с постоянной удвоения $\left(c_{2} / c_{1}\right) 2^{Q}$, см. определение 22 ниже. В свою очередь, это приводит к неравенству

$$
\lambda\left(G, d_{A}\right) \leqslant c\left(Q+\log _{2}\left(c_{2} / c_{1}\right)\right),
$$

где $c$ - числовая постоянная, см. теорему 25 ниже.

Б. Гиперболические групшы Громова. Чтобы ввести этот класс, нам нужны два основных понятия: граф Кәли и гиперболическое пространство Громова.

Чтобы определить второе из них, напомним, что метрическое пространство называется геодезическим, если любая пара его точек может быть соединена геодезическим сегментом. В свою очередь, геодезической сегмент, соединяющий $m$ и $m^{\prime}$, является образом такой кривой $\gamma:[0, a] \rightarrow M$, что $\gamma(0)=m, \gamma(a)=m^{\prime}$ и $d(\gamma(t), \gamma(s))=|t-s|$ для всех $t, s \in[0, a]$ (такая кривая $\gamma$ называется геодезической).

Например, риманово многообразие, снабженное геодезической метрикой (см. пример $7(б))$, является геодезическим метрическим пространством. То же верно для метрического дерева (метрического графа без циклов). Легко видеть, что геодезическое пространство является метрическим пространством путей.

ОПРЕДЕЛЕНИЕ 15. Геодезическое метрическое пространство $M$ назьвается $\delta$-гиперболическим пространством (Громова), $\delta \geqslant 0$, если любой геодезический треугольник $\delta$-тонок в том смысле, что любая сторона треугольника лежит в $\delta$-окрестности объединения остальных сторон. 
ПримеР 16 (подробности см., например, в [9]). (а) Любое полное односвязное риманово многообразие $M$, секционная кривизна $\kappa$ удовлетворяет условию $-b^{2}<\kappa<$ $-a^{2}<0$ для некоторых $a, b \in \mathbb{R}$, является гиперболическим по Громову. Из теоремы сравнения Рауха следует, что такое пространство $M$ есть также и пространство ограниченной геометрии, см., например, [14]. В частности, пространство Бельтрами-Лобачевского $\mathbb{H}^{n}$ - гиперболическое.

(б) Метрическое дерево 0-гиперболично. Это пространство - тоже пространство ограниченной геометрии, если степени его вершин равномерно ограничены.

(в) Рассматривая большие треугольники, нетрудно заметить, что евклидова плоскость $\mathbb{R}^{2}$ не гиперболична по Громову.

(г) Если $M$ квазиизометрично $M^{\prime}$ и $M^{\prime}$ гиперболично по Громову, то и $M$ тоже гиперболическое. Следовательно, $M$ не может быть гиперболическим, если оно содержит квазиизометрическую копию $\mathbb{R}^{2}$.

(д) Пусть $\Omega$ - сильно псевдовьпуклая область в $\mathbb{C}^{n}$ с границей класса $C^{2}$. В метрике Кобаяши, см. [25], оно становится гиперболическим по Громову, см. [4].

Пусть $G$ - группа с множеством образуюших $A$. Граф Кәли $C(G, A)$ пары $(G, A)$ есть метрический граф̆, определенный следуюшим образом. Элементы $g$ групшы $G-$ это вершины, а пары $(g, a g)$, где $a \in A \cup A^{-1},-$ ребра этого граффа. Согласованно отождествим ребра с интервалами единичной длины на числовой прямой. Граф Кэли связен: например, любой элемент $g \in G \backslash\{1\}$ может быть представлен в виде $g=a_{1} \cdots a_{l}$ при $a_{i} \in A \cup A^{-1}$, и поэтому 1 и $g$ соединены путем $1, a_{1}, a_{1} a_{2}, \ldots, g=a_{1} \cdots a_{l}$. Следовательно, определяя метрику $\widetilde{d}_{A}$ на $C(G, A)$ как в примере $7(\mathrm{a})$, мы превращаем граф Кэли в метрический граф. Тогда $\left(G, d_{A}\right)$ - метрическое подпространство $\left(C(G, A), \widetilde{d}_{A}\right)$ с метрикой, индуцированной $\widetilde{d}_{A}$. Естественные действия элементов $G$ на множестве вершин графа $C(G, A)$ могут быть продолжены до изометрий $\left(C(G, A), \widetilde{d}_{A}\right)$. Наконец, этот метрический граф - геодезическое пространство, подробности см. в $[9 ;$ с. 8]. Если между образуюшими $A$ есть нетривиальные соотношения, то в $C(G, A)$ есть циклы. В противном случае $G$ - свободная групn а с (возможно, бесконечным) множеством образуюших $A$. В этом случае мы используем обозначения $G:=\mathscr{F}(A)$ и $C(G, A):=C(\mathscr{F}(A))$. Так как этот граф не содержит циклов, то $\left(C(\mathscr{F}(A)), \widetilde{d}_{A}\right)$ - метрическое дерево.

Теперь мы готовы ввести упомянутое вьше основное понятие.

ОПРЕДЕЛЕНИЕ 17 . Конечно порожденная групп $G$ называется гиперболической (по Громову), если один из графов Кэли этой групшы является гиперболическим метрическим пространством. ${ }^{4}$

Мы будем также говорить, что такая группа $G \delta$-гиперболична, если можно выбрать такое множество образуюших $A$ в $G$, что графр Кэли $C(G, A)$ есть $\delta$-гиперболическое метрическое пространство.

ПримеР 18. (а) Конечные циклические групшы гиперболичны (они назьваются әлементарньми гиперболическими группами).

(б) Свободная группа конечного ранга 0-гиперболична.

(в) Дискретная кокомпактная группа изометрий пространства $\mathbb{H}^{n}$ гиперболична; в частности, фундаментальная группа компактной римановой поверхности рода $\geqslant 2$ гиперболична. При $n>2$ такие группы были построены А. Борелем [6] (арифметические групшы) и Громовым и Пятецким-Шапиро [20] (неарифометические групшы).

\footnotetext{
${ }^{4}$ В таком случае все граффы Кэли рассматриваемой группы имеют это свойство.
} 
(г) Более обшим образом, равномерная решетка (т.е. кокомпактная дискретная подгрупа) полупростой группы Ли ранга 1 гиперболична.

(д) Если $\Gamma$ - гиперболическая подгруппа конечного индекса в группе $G$, то $G$ гиперболична.

(е) Как заметил Громов [19], в некотором статистическом смысле почти любая конечно порожденная группа гиперболична, см. доказательство в статье Ольшанского $[32]$.

(ж) Если конечно порожденная группа $G$ содержит $\mathbb{Z}^{2}$ как подгруппу, то $G$ не гиперболична. В частности, прямое произведение бесконечных гиперболических групп не гиперболично. По той же причине равномерная решетка в полупростой группе Ли $G$ ранга > 1 не гиперболична (так как $G$ содержит изометрическую копию евклидовой плоскости как геодезическое подмногообразие).

(з) Гиперболическая група полиномиального роста либо конечна, либо виртуально циклична. Действительно, любая бесконечная гиперболическая группа, не являющаяся виртуально циклической, имеет экспоненциальньй рост.

ТЕОРема 19. Если $G$ - прямое произведение гиперболической группь и группь полиномиального роста, то $\lambda(G)<\infty$.

ЗАмЕчАнИЕ 20. В частности, если $G$ - дискретная кокомпактная группа изометрий $\mathbb{H}^{n}$, то орбита $G(m), m \in \mathbb{H}^{n}$, является равномерной решеткой экспоненциального роста с $\lambda(G(m))<\infty$ (см. определение перед гипотезой 12$)$.

Вернемся на время к гипотезе 12 для классов конечно порожденных групп, отличных от двух классов, рассмотренных выше. Простейшим является класс полициклических групп экспоненциального роста. По-видимому, в этом случае наши методы не работают; следовательно, было бы разумно исследовать проблему конечности величины $\lambda(G)$ для групп этого типа.

ЗАмечаниЕ 21. Теорема 19 есть следствие теорем 25 и 30, приведенных ниже. Как и эти два результата, теорема 19 справедлива для банаховозначных липшищевых функций.

Метрические пространства поточечно однородного типа. Чтобы ввести этот класс метрических пространств, нам нужно следующее

ОПРЕДЕЛЕНИЕ 22. Борелевская мера $\mu$ на метрическом пространстве $(M, d)$ называется мерой со свойством удвоения в точке $m \in M$, если любой открытьй шар с центром в $m$ имеет конечную строго положительную $\mu$-меру и постоянная удвоения

$$
D_{m}(\mu):=\sup _{R>0}\left\{\frac{\mu\left(B_{2 R}(m)\right)}{\mu\left(B_{R}(m)\right)}\right\}
$$

конечна.

Если, кроме того,

$$
D(\mu):=\sup _{m \in M} D_{m}(\mu)<\infty,
$$

то $\mu$ назьвается мерой со свойством удвоения.

Метрическое пространство, снабженное некоторой мерой со свойством удвоения, называется пространством однородного типа [16]. Заметим, что сушествуют метрические пространства, которые можно снабдить несчетньп множеством взаимно сингулярных мер со свойством удвоения [1]. Следовательно, пространство однородного типа есть тройка $(M, d, \mu)$. Как замечено в [16], пространство однородного типа есть 
метрическое пространство со свойством удвоения в том смысле, что любой замкнутый шар $\bar{B}_{2 R}(m)$ в этом пространстве можно покрыть не более чем $C^{\prime}$ шарами радиуса $R$, где $C^{\prime}$ не зависит от $R$. Оптимальная величина $C^{\prime}$ назьвается постоянной удвоения для $M$ (и обозначается $D(M)$ ). Эта постоянная удовлетворяет условию

$$
\log _{2} D(M) \leqslant c \log _{2} D(\mu)
$$

с числовой постоянной $c>1$. Здесь $\mu$-мера со свойством удвоения на $M$.

Наш основной класс метрических пространств вводится следуюшим образом.

ОПРЕДЕЛЕНИЕ 23. Метрическое пространство $(M, d)$ называется пространством поточечно однородного типа, если существует фиксированное семейство $\left\{\mu_{m}\right\}_{m \in M}$ борелевских мер на $M$, удовлетворяющих следующим условиям.

(i) Равномерное условие удвоения: $\mu_{m}$ имеет свойство удвоения в точке $m$ и

$$
D:=\sup _{m \in M} D_{m}\left(\mu_{m}\right)<\infty
$$

(ii) Совместимость с метрикой: для некоторой постоянной $C>0$ и всех $m_{1}, m_{2} \in M$ и $R>0$ имеем

$$
\left|\mu_{m_{1}}-\mu_{m_{2}}\right|\left(B_{R}(m)\right) \leqslant \frac{C \mu_{m}\left(B_{R}(m)\right)}{R} d\left(m_{1}, m_{2}\right),
$$

где $m=m_{1}$ или $m_{2}$.

ПримеР 24. (а) Метрическое пространство $(M, d, \mu)$ однородного типа, очевидно, принадлежит этому классу с $C=0$ и $D=D(\mu)$.

(б) Пусть $(M, d)$ - метрическое пространство со свойством удвоения и с постоянной удвоения $D(M)$. По теореме Вольберга-Конягина [34] (см. также [27] для некомпактного случая), $M$ несет такую меру $\mu$ со свойством удвоения, что

$$
\log _{2} D(\mu) \leqslant c \log _{2} D(M)
$$

где $c$ - числовая постоянная. Следовательно, $(M, d, \mu)$ - однородного типа и с постоянной $D(\mu)$, удовлетворяюшей условию (14).

(в) Пусть $\mathbb{H}_{\omega}^{n+1}$ - риманово многообразие на $\mathbb{R}_{+}^{n+1}:=\left\{(x, t) \in \mathbb{R}^{n} \times \mathbb{R}: t>0\right\}$ с римановой метрикой

$$
d s^{2}:=\frac{d x_{1}^{2}+\cdots+d x_{n}^{2}+d t^{2}}{\omega(t)^{2}} .
$$

Предположим, что $\omega$ непрерьвна и не убывает, а $t \mapsto \omega(t) / t^{2}$ не возрастает. Снабдим $\mathbb{H}_{\omega}^{n+1}$ семейством мер $\left\{\mu_{m}: m \in \mathbb{H}_{\omega}^{n+1}\right\}$, введенньм в [13]. Они определены на подмножествах $U \subset \mathbb{R}^{n} \times \mathbb{R}_{+}$по формуле

$$
\mu_{m}(U):=\int_{U} \chi(t-s) \widehat{\omega}(s) \prod_{i=1}^{n} \widehat{\omega}\left(t+\left|y_{i}-x_{i}\right|\right) d y_{1} \cdots d y_{n} d s
$$

Здесь $m=(x, t) \in \mathbb{R}^{n} \times \mathbb{R}_{+}$и

$$
\widehat{\omega}(t):=\frac{1}{\omega(t)}, \quad \chi(t):=\chi_{[0, \infty)}(t), \quad t \in \mathbb{R} .
$$


В [13; с. 537-540] было доказано, что это семейство мер удовлетворяет условиям определения 23 при $D=2^{n+1}$ и $C=6(n+1) 2^{n+1}$. Следовательно, $\mathbb{H}_{\omega}^{n+1}$, снабженное геодезической метрикой, является пространством поточечно однородного типа.

Следует заметить, что $\mathbb{H}_{\omega}^{n+1}$ совпадает с открытым полупространством евклидова пространства $\mathbb{R}^{n+1}$ при $\omega(t)=1, t>0$, и с гиперболическим пространством $\mathbb{H}^{n+1}$ при $\omega(t)=t, t>0$. Эти пространства имеют постоянную секционную кривизну. Однако это семейство содержит также пространства с неограниченной секционной кривизной (например, при $\omega(t)=t^{\alpha}$, где $0<\alpha \leqslant 2$ и $\alpha \neq 1$ ).

Список примеров можно продолжать, беря прямые суммы некоторых пространств поточечно однородного типа, см. определение 27 ниже.

Приведем теперь наш основной результат о пространствах поточечно однородного типа (доказательства см. в [10; теорема 2.22] в скалярном случае и в [12] для функций со значениями в банаховом пространстве).

TеОРема 25. Пусть $M$ пространство поточечно однородного типа с оптимальными постоянными $C$ и $D$ (см. определение 23). Тогда для любого (не обязательно сопряженного) банахова пространства $X$ имеем

$$
\lambda(M, X) \leqslant k_{0}(C+1)\left(\log _{2} D+1\right) ;
$$

здесь $k_{0}-$ числовая постоянная.

ЗАмЕчАНИЕ 26. Для метрического пространства $(M, d, \mu)$ однородного типа величина $D$ больше, чем, скажем, $\sqrt[4]{2}$ (см., например, [16]), и из теоремы 25 следует, что

$$
\lambda(M, X) \leqslant 2 k_{0} \log _{2} D(\mu) .
$$

Это неравенство можно вывести также из теоремы 1.6 статьи Ли и Наора [26]. Их результат утверждает, что

$$
\lambda(S, M, X) \leqslant c_{0} \log _{2} D(S),
$$

если $S$ - метрическое подпространство со свойством удвоения в произвольном метрическом пространстве $M$. Действительно, если само $M$ является метрическим пространством со свойством удвоения с постоянной удвоения $D(M)$, то из предыдущего неравенства следует, что

$$
\lambda(M, X) \leqslant c_{0} \log _{2} D(M) .
$$

Тогда применение неравенства (13) завершает вьвод.

Следует заметить, что теорема Ли-Наора допускает конструктивное доказательство, подобное доказательству теоремы 25. Было бы интересно найти вариант неравенства (15) с пространством $S, C$-изометричньм подпространству метрического пространства $M_{0}$ поточечно однородного типа, и с правой частью, зависяшей только от $C$ и от оптимальных постоянных для $M_{0}$. По-видимому, это может потребовать дополнительных условий на $M$. Однако результат этого типа справедлив, например, для $S$, $C$-изометричного (метрическому) подпространству гиперболического пространства $M^{\prime}:=\mathbb{H}^{n}$, и для произвольного $M$ (см. теорему 32 ниже).

Чтобы сформулировать некоторые следствия теоремы 25 , нам нужно следующее определение. 
ОПРЕДЕЛЕНИЕ 27. Семейство борелевских мер $\left\{\mu_{m}\right\}_{m \in M}$ на метрическом пространстве $M$ назьвается $K$-равномерным $(K \geqslant 1)$, если для любых $m_{1}, m_{2}$ и $R>0$ имеем

$$
\mu_{m_{1}}\left(B_{R}\left(m_{1}\right)\right) \leqslant K \mu_{m_{2}}\left(B_{R}\left(m_{2}\right)\right)
$$

Заметим, что для 1-равномерного семейства величина $\mu_{m}\left(B_{R}(m)\right)$ не зависит от $m$. В частности, если для всех шаров в $M$ вьполняется условие

$$
\mu_{m}\left(B_{R}(m)\right)=\gamma R^{n}, \quad \gamma, n>0,
$$

и если $M$ удовлетворяет условиям определения 23 , то $M$ называется пространством поточечно $n$-однородного типа. Заметим, что в этом случае $D=2^{n}$, в то время как $C \geqslant 0$ может быть любым.

СлЕДСТВИЕ 28 [10; следствие 2.26]. Пусть $M-$ пространство поточечно n-однородного типа с оптимальной постоянной $C$. Тогда

$$
\lambda(M, X) \leqslant 24(n+C) .
$$

Пример 29. Группа Карно (известная также как однородная группа), см., например, [18], - это односвязная вешественная группа Ли $G$, чья алгебра Ли $g$ допускает стратификацию

$$
g=\bigoplus_{i=1}^{m} V_{i} \quad \text { c }\left[V_{i}, V_{i}\right]=V_{i+1}
$$

здесь $V_{m+1}=\{0\}$ и $V_{m} \neq\{0\}$.

Будучи нильпотентной, $G$ диффеоморфна $\mathbb{R}^{n}$ с $n:=\operatorname{dim} G$. Наряду с топологической размерностью $n$, важную роль играет однородная размерность $G$, определяемая формулой

$$
\operatorname{dim}_{h} G:=\sum_{j=1}^{m} j \operatorname{dim} V_{j} .
$$

Группа $G$ может быть снабжена левоинвариантной метрикой $d$ (метрикой $K a p н o-K a-$ paтеодори), для которой отображение $B_{r}(x):=\{y \in G: d(x, y)<r\}$ удовлетворяет условию

$$
\left|B_{r}(x)\right|=r^{Q}, \quad x \in G, \quad r>0 .
$$

Символ $|\cdot|$ означает здесь (нормированную) левоинвариантную меру Хаара на $G$ и $Q:=\operatorname{dim}_{h} G$. Поэтому из следствия 28 непосредственно получаем

$$
\lambda(G, d) \leqslant 24 \operatorname{dim}_{h} G .
$$

Простейший пример группы Карно - это $\mathbb{R}^{n}$. В этом случае $\operatorname{dim}_{h} G=\operatorname{dim} G=n$, и любая банахова норма на $\mathbb{R}^{n}$ определяет метрику Карно-Каратеодори. Это дает упомянутую выше теорему продолжения статьи [21] с улучшенной постоянной.

Другой интересньй пример групшы Карно - группа Гейзенберга $H_{n}(\mathbb{R})$, которая совпадает с $\mathbb{R}^{n} \times \mathbb{R}^{n} \times \mathbb{R}$ как множество. Групповая операция определяется по формуле

$$
(x, y, t) \cdot\left(x^{\prime}, y^{\prime}, t^{\prime}\right)=\left(x+x^{\prime}, y+y^{\prime}, t+t^{\prime}+\left\langle x^{\prime}, y\right\rangle\right)
$$

где первые две координаты - векторы в $\mathbb{R}^{n}$, а $\langle\cdot, \cdot\rangle$ - стандартное скалярное произведение. Топологическая размерность пространства $H_{n}(\mathbb{R})$, очевидно, равна $2 n+1$, 
в то время как его однородная размерность равна $2 n+2$. Наконец, метрика Карно-Каратеодори $d$ определяется формулой

$$
d\left((x, y, t),\left(x^{\prime}, y^{\prime}, t^{\prime}\right)\right):=\left|(x, y, t)^{-1} \cdot\left(x^{\prime}, y^{\prime}, t^{\prime}\right)\right|,
$$

где $|(x, y, t)|:=\left(\langle x, x\rangle^{2}+\langle y, y\rangle^{2}+t^{2}\right)^{1 / 4}$.

Для метрического пространства $\left(H_{n}(\mathbb{R}), d\right)$ неравенство $(19)$ дает верхнюю границу $\lambda\left(H_{n}(\mathbb{R})\right) \leqslant 48(n+1)$. Интересно отметить, что метод продолжения Уитни не работает даже для доказательства неравенства $\lambda\left(H_{n}(\mathbb{R})\right)<\infty$. Действительно, его основная геометрическая составляющая, лемма Уитни о покрытии, не может быть доказана в такой постановке в форме, допускающей требуемое липшицево разложение единицы.

Пусть теперь $(M, d):=\bigoplus_{p}\left\{\left(M_{i}, d_{i}\right)\right\}_{1 \leqslant i \leqslant N}$ - прямая $p$-сумма метрических пространств $\left(M_{i}, d_{i}\right)$, т.е. метрическое пространство на множестве $M=\prod_{i=1}^{N} M_{i}$ с метрикой $d$, определенной формулой

$$
d\left(m, m^{\prime}\right):=\left(\sum_{i=1}^{N} d_{i}\left(m_{i}, m_{i}^{\prime}\right)^{p}\right)^{1 / p}
$$

здесь $m=\left(m_{1}, \ldots, m_{N}\right), m^{\prime}=\left(m_{1}^{\prime}, \ldots, m_{N}^{\prime}\right)$ и $1 \leqslant p \leqslant \infty$.

Особенно интересны случаи $p=1, \infty$. Первьй их них возникает, например, при определении расстояния между словами в прямом произведении конечно порожденных груп, в то время как второй случай возникает при определении метрики Кобаяши на прямом произведении гиперболических комплексных многообразий Кобаяши [25].

Теорема 30 [10; теорема 2.28]). Пусть $M_{i}$ - пространство поточечно однородного типа относительно $K_{i}$-равномерного семейства борелевских мер $\left\{\mu_{m}^{i}\right\}_{m \in M_{i}}$ удовлетворяющих условиям определения 23 с оптимальными постоянными $D_{i}, C_{i}, 1 \leqslant i \leqslant N$. Тогда для любого банахова пространства $X$ выполняется неравенство

$$
\lambda\left(\bigoplus_{p}\left\{\left(M_{i}, d_{i}\right)\right\}_{1 \leqslant i \leqslant N} ; X\right) \leqslant c_{0}\left(\widetilde{C}_{p}+1\right)\left(\log _{2} \widetilde{D}+1\right)
$$

здесь

$$
\widetilde{D}:=\prod_{i=1}^{N} D_{i}, \quad \widetilde{C}_{p}:=\left(\sum_{i=1}^{N} C_{i}^{q}\right)^{1 / q}\left(\prod_{i=1}^{N} K_{i}\right)
$$

где $c_{0}-$ числовая постоянная и $q$ - показатель, сопряженный $к p\left(\right.$ m.е. $\left.\frac{1}{p}+\frac{1}{q}=1\right)$.

Если $\left(M_{i}, d_{i}\right)$ - пространства поточечно $n_{i}$-однородного типа, $1 \leqslant i \leqslant N$, то оценку теоремы 30 можно уточнить. Например, справедливо неравенство

$$
\lambda\left(\bigoplus_{\infty}\left\{\left(M_{i}, d_{i}\right)\right\}_{1 \leqslant i \leqslant N}\right) \leqslant 24 \sum_{i=1}^{N}\left(n_{i}+C_{i}\right) .
$$

Иногда оказывается возможным получить нижнюю гранищу для постоянной продолжения для прямых p-сумм теоремы 30 (см., например, неравенство (22) ниже для 
случая прямых $p$-сумм метрических деревьев с $p=1, \infty)$. Другой пример - прямая 2 -сумма семейства $\left\{\left(R_{i}, d_{g_{i}}\right)\right\}_{1 \leqslant i \leqslant N}$ римановых многообразий, для которых из теоремы 4 (б) следует, что

$$
c_{0}\left(\sum_{i=1}^{N} \operatorname{dim} R_{i}\right)^{1 / 8} \leqslant \lambda\left(\bigoplus_{2}\left\{\left(R_{i}, d_{g_{i}}\right)\right\}_{1 \leqslant i \leqslant N}\right) .
$$

Чтобы сформулировать следующий результат, воспользуемся еще одним определением.

ОПРЕДЕЛЕНИЕ 31 . Метрическое пространство $\left(M_{0}, d_{0}\right)$ назьвается универсальнылм относительно совместного липшицева продолжения, если для любого метрического пространства $(M, d)$ и любого подпространства $S$ в $M$, квазиизометричного подпространству $\left(M_{0}, d_{0}\right)$, вьполняется неравенство

$$
\sup _{X} \lambda(S, M, X)<\infty
$$

здесь верхняя грань берется по всем банаховым пространствам $X$.

В качестве примера универсального метрического пространства можно взять, например, метрическое пространство со свойством удвоения. Действительно, по упомянутой вьше теореме Ли-Наора (см. замечание 26), если метрическое пространство со свойством удвоения $M_{0}$ является подпространством произвольного метрического пространства $M$, то

$$
\sup _{X} \lambda\left(M_{0}, M, X\right) \leqslant K_{0} \log _{2} D\left(M_{0}\right) .
$$

Так как любое подпространство $S$ пространства $M_{0}$ наследует свойство удвоения и $D(S) \leqslant D\left(M_{0}\right)$, последнее неравенство устанавливает универсальность пространства $M_{0}$ (в смысле определения 31 ). Рассмотрим теперь примеры универсальных метрических пространств, не имеющих подобных наследственных свойств.

Tеорема 32 [12]. Пусть $(\widetilde{M}, \widetilde{d}):=\bigoplus_{p}\left\{\left(M_{i}, d_{i}\right)\right\}_{1 \leqslant i \leqslant N}$, где любое слагаемое $\left(M_{i}, d_{i}\right)$ является либо гиперболическим пространством (по Громову) ограниченной геометрии либо метрическим пространством с условием удвоения. Тогда $(\widetilde{M}, \widetilde{d})$ универсально.

ЗАмЕчАнИЕ 33. Иногда нужно оценить величину $\lambda(S, M, X)$ для произвольного метрического пространства $M$ и его подпространства $S, C$-изометричного подпространству пространства $\widetilde{M}$, с помощью постоянной, зависяшей только от $\widetilde{M}$ и $C$. В доказательствах мы установили такие оценки для широкого класса пространств $\widetilde{M}$. Например, если $M_{i}$ - гиперболическое пространство $\mathbb{H}^{n_{i}}, 1 \leqslant i \leqslant N$, а подпространство $S$ метрического пространства $M C$-изометрично подпространству $\bigoplus_{1}\left\{\mathbb{H}^{n_{i}}\right\}_{1 \leqslant i \leqslant N}$, то для любого банахова пространства $X$ получаем

$$
\lambda(S, M, X) \leqslant c C^{2} N\left(\max _{1 \leqslant i \leqslant N} n_{i} 2^{n_{i}}+\sum_{i=1}^{N} n_{i}\right),
$$

где $c$ - числовая постоянная.

Среди составляющих доказательства теоремы 32 следующий геометрический факт интересен сам по себе. 
ТеОрема 34 [10; предложение 7.1]. Любое бесконечное метрическое дерево с равномерно ограниченными степенями вершин допускает квазиизометрическое вложение в гиперболическую плоскость $\mathbb{H}^{2}$ с искажением ${ }^{5}$, ограниченным некоторой числовой постоянной.

Кажется более естественным использовать здесь евклидово пространство вместо гиперболического пространства. Удивительно, но этого нельзя добиться, даже если использовать бесконечномерное гильбертово пространство, см. статью Бургейна [8].

Заметим, что метрическое дерево, удовлетворяющее условиям теоремы 34 , есть 0-гиперболическое пространство ограниченной геометрии. В частности, конечная прямая сумма таких метрических деревьев универсальна. Этот факт можно усилить, если ограничиться $X$-значными липшицевьми функциями со значениями в сопряженном пространстве $X$. А именно, справедливо следующее утверждение.

Теорема $35\left[10\right.$; теорема 2.5]. Пусть $(\widetilde{M}, \widetilde{d}):=\bigoplus_{p}\left\{\left(\mathscr{T}_{i}, d_{i}\right)\right\}_{1 \leqslant i \leqslant N}$, әде $\left(\mathscr{T}_{i}, d_{i}\right)$ - произвольнье метрические деревья. Тогда для любого метрического пространства $(M, d)$ и любого подпространства $S(\subset M)$, квазиизометричного подпространству $(\widetilde{M}, \widetilde{d})$, имеем

$$
\sup _{X} \lambda\left(S, M, X^{*}\right)<\infty
$$

здесь верхняя грань берется по всем банаховьм пространствам $X$.

В частности, для нетривиальных метрических деревьев $\mathscr{T}_{i}$ и при $p=1, \infty$ мы получаем доказательство неравенства

$$
c_{0} \sqrt{N} \leqslant \sup _{X} \lambda\left(\bigoplus_{p}\left\{\left(\mathscr{T}_{i}, d_{i}\right)\right\}_{1 \leqslant i \leqslant N}, X^{*}\right) \leqslant c_{1} N,
$$

где $c_{0}$ и $c_{1}$ - числовые постоянные.

Как и выше, пусть $\mathscr{F}(A)$ означает свободную групшу с (не обязательно конечньм) множеством образуюших $A$ и с расстоянием между словами $d_{A}$. Тогда метрическое пространство $(M, d):=\bigoplus_{1}\left\{\left(\mathscr{F}\left(A_{i}\right), d_{A_{i}}\right)\right\}_{1 \leqslant i \leqslant N}$, как множество, есть группа $\prod_{i=1}^{N} \mathscr{F}\left(A_{i}\right)$ (с множеством образующих $\prod_{i=1}^{N} A_{i}$ ). Расстояние между словами в этой групе совпадает с метрикой $d$ прямой суммы. Кроме того, граф Кэли имеет вид $C\left(\prod_{i=1}^{N} \mathscr{F}\left(A_{i}\right), \widetilde{d}_{\prod_{i=1}^{N} A_{i}}\right)=\bigoplus_{1}\left\{\left(C\left(\mathscr{F}\left(A_{i}\right)\right), \widetilde{d}_{A_{i}}\right)\right\}_{1 \leqslant i \leqslant N}$, и любой множитель справа является метрическим деревом. Отсюда и из (22) непосредственно следуют неравенства

$$
c_{0} \sqrt{N} \leqslant \lambda\left(\bigoplus_{1}\left\{\left(\mathscr{F}\left(A_{i}\right), d_{A_{i}}\right)\right\}_{1 \leqslant i \leqslant N}\right) \leqslant c_{1} N .
$$

Основные результаты. Приведем теперь несколько общих утверждений, применение которых приводит к результатам предыдущей части статьи.

A. Свойство конечности. Чтобы сформулировать результат, определим дилатацию метрического пространства $(M, d)$ как такую квазиизометрию $\delta$ пространства $M$ на себя, что оператор $\Delta: \operatorname{Lip}(M) \rightarrow \operatorname{Lip}(M)$, заданный формулой

$$
(\Delta f)(m):=f(\delta(m)), \quad m \in M,
$$

удовлетворяет условию

$$
\|\Delta\| \cdot\left\|\Delta^{-1}\right\|=1
$$

\footnotetext{
${ }^{5}$ Напомним, что искажсение квазиизометрии $\phi: M_{1} \rightarrow M_{2}$ метрических пространств определяется формулой $|\phi|_{\operatorname{Lip}\left(M_{1}, M_{2}\right)} \cdot\left|\phi^{-1}\right|_{\operatorname{Lip}\left(M_{2}, M_{1}\right)}$.
} 
Теорема 36 [10; теорема 2.1]. Предположим, что $S$ - такое подпространство $M$, что для некоторой дилатации $\delta: M \rightarrow M$ имеем

(i) $S \subset \delta(S)$;

(ii) $\bigcup_{j \geqslant 0} \delta^{j}(S)$ плотно в $M$.

Тогда

$$
\lambda(M)=\sup _{F \subset S} \lambda(F)
$$

где F пробегает все подпространства $S$ с конечным числом точек.

Полагая $S=M$ и беря тождественное отображение в качестве $\delta$, получаем отсюда следующее утверждение.

СЛЕДСТВИЕ 37.

$$
\lambda(M)=\sup _{F} \lambda(F),
$$

где F пробегает все подпространства $S$ с конечным числом точек.

Отсюда, в сочетании с теоремой 36 , вытекает

СлЕДСТВИЕ 38. Пусть $S \subset M$ удовлетворяет условиям теоремы 36 . Тогда

$$
\lambda(M)=\lambda(S) .
$$

$\mathrm{B}$ качестве простого примера рассмотрим целочисленную решетку $\mathbb{Z}^{n}$ как метрическое подпространство в $l_{1}^{n}$ (или, что то же, как абелеву группу с расстоянием между словами, определенным стандартньм базисом в $\left.\mathbb{R}^{n}\right)$. Тогда

$$
\lambda\left(\mathbb{Z}^{n}\right)=\lambda\left(l_{1}^{n}\right),
$$

откуда следует, что (см. теорему 4 ) для некоторого $c>0$ и для всех $n$ имеем

$$
c \sqrt{n} \leqslant \lambda\left(\mathbb{Z}^{n}\right) \leqslant 24 n .
$$

Это неравенство играет важную роль в построении контрпримера в теореме 9.

Б. Нелинейные и совместные липшищевы продолжения. Следуюший результат связывает нелинейную постоянную продолжения $\nu(M, X)$ с $\lambda(M)$ (см. (6) по поводу определения $\nu(M, X))$.

TeOpema 39 [11].

$$
\lambda(M)=\sup _{X} \nu(M, X),
$$

где $X$ пробегает все конечномерные банаховы пространства.

B. Слабые и сильные совместные лишшищевы продолжения. Мы будем говорить, что метрическое пространство $M$ имеет слабое свойство совместного липшищева продолжсения (и писать $M \in \mathscr{W} L E$ ), если для любого подпространства $S$ имеем

$$
\lambda(S, M)<\infty .
$$

Вопрос о том, следует ли из этого свойства конечность величины $\lambda(M)$, открыт. (Мы полагаем, что ответ отрищателен.) Следуюший результат дает достаточные условия справедливости этой импликации. В его формулировке мы используем следующее определение.

ОПРЕДЕЛЕНИЕ 40. (а) Метрическое пространство $M$ назьвается собственным (или ограниченно компактным), если любой замкнутый шар в $M$ компактен. 
(б) Метрическое пространство $M$ имеет свойство перехода (ТР), если для некоторого $C \geqslant 1$, любого конечного множества $F$ и любого открытого шара $B$ в $M$ сушествует такая $C$-изометрия $\sigma: M \rightarrow M$, что

$$
B \cap \sigma(F)=\varnothing
$$

Теорема 41 [10; теорема 2.7]. Предположим, что $M$ либо является собственным, либо имеет свойство перехода. Неравенство $\lambda(M)<\infty$ выполняется тогда и только тогда, когда $M \in \mathscr{W} L E$.

ЗАмЕчАниЕ 42 . Объединяя этот результат с теоремой 36 , можно в некоторых случаях упростить вывод оценки для $\lambda(M)$ для метрических пространств из определения 40. А именно, сначала можно доказать для данного подпространства $S \subset M$ конечность величины $\lambda(S, M)$ с любой оценкой и затем применить теорему 36 , чтобы выбрать $S$ для получения оценки величины $\lambda(M)$.

Г. Метрические пространства со свойством локального удвоения.

ОПРЕДЕЛЕНИЕ 43. Метрическое пространство $M$ назьвается пространством со свойством локального удвоения, если для некоторого $R>0$ и некоторого целого $N$ любой шар радиуса $r<R$ может быть покрыт не более чем $N$ шарами радиуса $r / 2$.

Обозначим класс таких пространств символом $\mathscr{D}(R, N)$.

Следующий результат лежит в основе доказательств теорем 11 и 13.

Tеорема 44 [10; теорема 2.10]. Пусть $M \in \mathscr{D}(R, N)$. Пусть $\Gamma-R$-решетка в $M$, см. определение 10. Предположим, что постоянные $\lambda(\Gamma)$ и

$$
\lambda_{R}:=\sup _{m \in M}\left\{\lambda\left(B_{R}(m)\right)\right\}
$$

конечны. Тогда $\lambda(M)$ ограничена постоянной, явно зависящей от $\lambda(\Gamma), \lambda_{R}, R$ $u N$.

\section{СПИСОК ЛИТЕРАТУРЫ}

[1] L. Ahlfors, A. Beurling. The boundary correspondence under quasiconformal mappings // Acta Math. 1956. V. 96. P. 125-142.

[2] N. Aronszajn, N. Panitchpakdi. Extension of uniformly continuous transformations in hyperconvex metric spaces // Pacific J. Math. 1956. V. 6. P. 405-439; correction: ibid. 1957. V. 7. P. 1729.

[3] K. Ball. Markov chains, Riesz transforms and Lipschitz maps // Geom. Funct. Anal. 1992. V. 2. № 2. P. 137-172.

[4] Z. M. Balogh, M. Bonk. Gromov hyperbolicity and the Kobayashi metric on strictly pseudoconvex domains // Comment. Math. Helv. 2000. V. 75. №3. P. 504-533.

[5] H. Bass. The degree of polynomial growth of finitely generated groups // Proc. London Math. Soc. (3). 1972. V. 25. P. 603-614.

[6] A. Borel. Compact Clifford-Klein forms of symmetric spaces // Topology. 1963. V. 2. № 2 . P. 111-122.

[7] K. Borsuk. Über Isomorphie der Funktionalräume // Bull. Int. Acad. Polon. Sci. A. 1933. № $1 / 3$. P. 1-10.

[8] J. Bourgain. The metrical interpretation of superreflexivity in Banach spaces // Israel J. Math. 1986. V. 56. № 2. P. 222-230.

[9] M. Bridson, A. Haefliger. Metric Spaces of Non-positive Curvature. Berlin: SpringerVerlag, 1999. (Grundlehren Math. Wiss. V. 319.) 
[10] A. Brudnyi, Yu. Brudnyi. Metric spaces with linear extensions preserving Lipschitz condition // Amer. J. Math. (to appear); http://arxiv.org: math.MG/0404304.

[11] A. Brudnyi, Yu. Brudnyi. Linear and nonlinear extensions of Lipschitz functions from subsets of metric spaces // Studia Math. (to appear).

[12] A. Brudnyi, Yu. Brudnyi. A universal Lipschitz extension property for products of Gromov hyperbolic spaces // Preprint. Univ. of Calgary, 2005.

[13] Yu. Brudnyi, P. Shvartsman. The Whitney problem of existence of a linear extension operator // J. Geom. Anal. 1997. V. 7. № 4. P. 515-574.

[14] J. Cheeger, D. G. Ebin. Comparison Theorems in Riemannian Geometry. Amsterdam: North-Holland, 1975. (North-Holland Math. Library. V. 9.)

[15] J. Cheeger, M. Gromov. Bounds of the von Neumann dimension of $L^{2}$-cohomology and the Gauss-Bonnet theorem for open manifolds // J. Differential Geom. 1985. V. 21. № 1. P. $1-34$.

[16] R. R. Coifman, G. Weiss. Analyse harmonique non-commutative sur certains espaces homogènes. Berlin: Springer-Verlag, 1971. (Lecture Notes in Math. V. 242.)

[17] J. Dugundji. An extension of Tietze's theorem // Pacific J. Math. 1951. V. 1. P. 353-367.

[18] G. B. Folland, E. M. Stein. Hardy Spaces on Homogeneous Groups. Princeton: Princeton Univ. Press, 1982. (Math. Notes. V. 28.)

[19] M. Gromov. Hyperbolic groups // Essays in Group Theory / ed. S. M. Gersten, 1987. P. 75-263. (Math. Sci. Res. Inst. Publ. V. 8.)

[20] M. Gromov, I. Piatetski-Shapiro. Nonarithmetic groups in Lobachevsky spaces // Inst. Hautes Études Sci. Publ. Math. 1988. V. 66. P. 93-103.

[21] W. B. Johnson, J. Lindenstrauss, G. Schechtman. Extensions of Lipschitz maps into Banach spaces // Israel J. Math. 1986. V. 54. № 2. P. 129-138.

[22] S. Kakutani. Simultaneous extension of continuous functions considered as a positivelinear operation // Japan. J. Math. 1940. V. 17. P. 1-4.

[23] Б. С. Кашин. Поперечники некоторых конечномерных множеств и классов гладких функций // Изв. АН СССР. Сер. матем. 1977. Т. 41. № 2. С. 334-351.

[24] M.D. Kirszbraun. Über die zusammenziehende und Lipschitsche Transformationen // Fund. Math. 1934. V. 22. P. 77-108.

[25] S. Kobayashi. Hyperbolic Manifolds and Holomorphic Mappings. New York: Dekker, 1970. (Pure Appl. Math. V. 2.)

[26] J.R. Lee, A. Naor. Extending of Lipschitz functions via random metric partitions // Invent. Math. 2005. V. 160. № 1. P. 59-95.

[27] J. Luukkainen, E. Saksman. Every complete doubling metric space carries a doubling measure // Proc. Amer. Math. Soc. 1998. V. 126. № 2. P. 531-534.

[28] M. B. Markus, G. Pisier. Characterization of almost surely continuous $p$-stable random Fourier series and strongly stationary processes // Acta Math. 1984. V. 152. № 3-4. P. 245-301.

[29] J. Matoušek. Extension of Lipschitz mappings on metric trees // Comment. Math. Univ. Carolin. 1990. V. 31. № 1. P. 99-104.

[30] E. J. McShane. Extension of range of functions // Bull. Amer. Math. Soc. 1934. V. 40. № 12. P. $837-842$.

[31] A. Naor. A phase transition phenomen between isometric and isomorphic extension problems for Hölder functions between $L_{p}$ spaces // Mathematika. 2001. V. 48. № 1-2. P. 253-271.

[32] A. Yu. Ol'shanskii. Almost every group is hyperbolic // Internat. J. Algebra Comput. 1992. V. 2. № 1. P. 1-17.

[33] A. Pełczyński. Linear extensions, linear averagings, and their applications to linear topological classification of spaces of continuous functions // Dissertationes Math. (Rozprawy Mat.). 1968. V. 58.

[34] А. Л. Вольберг, С. В. Конягин. На любом компакте в $\mathbb{R}^{n}$ существует однородная мера // Докл. АН СССР. 1984. Т. 278. № 4. С. 783-786.

[35] H. Whitney. Analytic extensions of differentiable functions defined in closed sets // Trans. Amer. Math. Soc. 1934. V. 36. P. 63-89.

University of Calgary, Canada;

Technion, Haifa, Israel

Поступила в редакцию 30.09 .2005 